Agata ZAPŁOTNA ${ }^{1}$

Uniwersytet Wrocławski

\title{
La vitalità del congiuntivo nel linguaggio televisivo
}

\begin{abstract}
Summary
The purpose of the article is to examine the relationship between television language and spoken Italian, applying the problem of the subjunctive as a reference point, frequently replaced by the indicative in colloquial contexts. Despite the fact that television language is now considered a reflection of Italian varieties and spoken-spoken language, there is still a heterogeneous language of the type transmitted. As such, it constitutes a set of various styles and registers, characterized by different linguistic phenomena from those of unattended speech.

The effect of the study is to show that although in the field of Italian diametric varieties television language has more in common with spoken than written Italian, the dynamics of the processes that take place in both are still not the same.
\end{abstract}

Keywords: media language, spoken language, Italian

Lo scopo dell'articolo è quello di esaminare il rapporto tra il linguaggio televisivo e l'italiano parlato, applicando come punto di riferimento il problema del congiuntivo, frequentemente sostituito dall'indicativo nei contesti colloquiali. Nonostante che il linguaggio televisivo venga oggi considerato un rispecchiamento delle varietà italiane e del parlato-parlato, rimane ancora una eterogenea lingua del tipo trasmesso. Come tale, costituisce un insieme dei vari stili e registri, caratterizzati per diversi fenomeni linguistici da quelli del parlato non sorvegliato.

La ricerca è basata sull'analisi quantitativa dei due corpora: uno del linguaggio televisivo (LIT) e l'altro dell'italiano parlato (CorDIC), nei quali vengono ricercati verbi inclini all'alternanza dei modi. In risultato è stata scoperta una significativa sproporzione tra l'uso del congiuntivo in entrambe le banche dati.

L'effetto dello studio è dimostrare che sebbene nel campo delle varietà diamesiche italiane il linguaggio televisivo abbia più in comune con italiano parlato che scritto, la dinamica dei processi che si svolgono in entrambi ancora non è uguale.

1 (D) https://orcid.org/0000-0001-6013-827X. 


\section{Introduzione}

Luca Serianni nella sua Prima lezione di grammatica accerta che il linguaggio televisivo "ha dato largo spazio al parlato reale ed è diventato piuttosto rispecchiamento che modello di lingua"2. Tuttavia, nonostante che il linguaggio televisivo abbia indubbiamente contribuito alla diffusione e l'unificazione dell'italiano ${ }^{3}$, una simile tesi sembra piuttosto esagerata e generalizzante. Il parlato della televisione riflette, difatti, un vasto panorama delle varietà linguistiche, raccogliendo tutto il "continuum dell'italiano nel piccolo spazio linguistico" ${ }^{4}$, ma rimane sempre una variante specifica del parlato trasmesso, che possiede caratteristiche del parlato-scritto, vale a dire è "scritto per essere detto come se non fosse scritto"5. Costituisce quindi un complesso misculio di diversi stili e registri, usato sia nei telegiornali e film doppiati sia nei reality shows, dove ognuno dei contesti influisce sulla lingua parlata non sorvegliata.

Tenendo in considerazione la complessità ed eterogeneità della lingua dei mass media, con il presente studio si cerca di verificare se il linguaggio televisivo rispecchia per davvero le strategie linguistiche adoperate in una conversazione spontanea. Come criterio d'analisi viene proposta l'alternanza dei modi congiuntivo e indicativo negli stessi contesti sintattici, considerata come una delle caratteristiche dell'italiano parlato ${ }^{6}$. Le analisi quantitative sono state effettuate sui copora LIT (Linguaggio italiano televisivo) e Cordic (Corpora Didattici Italiani di Confronto) e vogliono dimostrare in che misura il linguaggio televisivo assomiglia al parlato non sorvegliato per quanto riguarda l'uso del congiuntivo: è un vero e proprio "rispecchiamento" o forse è più vicino al parlato-scritto, caratterizzato da un grado moderatamente basso di spontaneità.

\section{Il linguaggio televisivo}

Il periodo cruciale per lo svliluppo dell'italiano contemporaneo, qundi anche della sua variante trasmessa, sono gli anni Settanta, quando tutte le varietà italiane,

\footnotetext{
2 L. Serianni, Prima lezione di grammatica, Roma 2011, p.246.

${ }^{3}$ R. Setti, Primi sondaggi sul lessico televisivo dei programmi RAI, [in:] I linguaggi dei media, a cura di S. Stefanelli, V. Saura, Firenze 2012, p. 131.

${ }^{4}$ T. De Mauro, Storia linguistica dell'Italia Unita, Bari 1979, p. 439.

5 C. Lavinio, Fiaba popolare, oralità, vernacolo, [in:] Interni e dintorni del Pinocchio. Atti del Convegno Folkloristi italiani del tempo del Collodi, a cura di P. Clemente e M. Festa, Montepulciano 1986, p. 16.

${ }^{6}$ Ramat 1983, Berruto 1993, Serianni 2006.
} 
inclusi i dialetti, vengono accettati nella produzione dei programmi televisi ${ }^{7}$. Vista una nuova apertura a ogni tipo di parlante e la presenza di diverse varietà regionali (grazie ad esempio alle trasmissioni in diretta), il linguaggio televisivo perde il suo ruolo educativo (non funge più da modello di lingua) per rispecchiare, d'ora in poi, la vera realtà linguistica italiana ${ }^{8}$. In tal modo cresce l'autenticità del parlato televisivo, ma - allo stesso tempo - si impoverisce la lingua stessa9 ${ }^{9}$. I mass media tendono, infatti, a "riprodurre gli usi medi e comuni" della lingua ${ }^{10}$, influendo sulle abitudini linguistiche e contribuendo quindi all'evoluzione dell'italiano contemporaneo.

Il linguaggio televisivo, considerato per il suo sincretismo "[...] una sintesi tra l'oralità della radio e l'iconicità del cinema"11, costituisce un punto di riferimento affidabile per l'analisi del parlato ${ }^{12}$. Infatti, è una lingua trasmessa (parlato-scritto) e come tale combina i tratti del parlato e dello scritto. Da una parte, il messaggio è riproducibile e conservabile, non c'è la comunicazione diretta tra il mittente e l'emittente, che regola la trasmissione. Dall'altra parte, dimostra tutti i tratti tipici per il parlato riprodotto: il sistema pronominale ridotto, usi verbali colloquiali, dislocazioni e frasi scisse, paratassi e subordinazione scarsa ${ }^{13}$. Il mezzo e il canale della trasmissione fanno sì sia pressoché impossibile considerare la lingua televisiva un parlato non sorvegliato, anche se - ovviamente - il grado della sua spontaneità dipende sempre dal tipo del programma ${ }^{14}$. Considerando il mezzo, non si tratta, in questo caso, del parlato del tutto spontaneo e la lingua dei mass media non può però essere vista come un insieme omogeneo e unitario ${ }^{15}$.

Giacché, come si è detto sopra, lo stesso Serianni ha definito il linguaggio televisivo come specchio dei processi che si svolgono nel parlato, vale la pena come crediamo - analizzare in che misura viene ivi impiegato il congiuntivo, quindi uno dei problemi più vistosi relativi al parlato. Con la nostra ricerca

\footnotetext{
7 P. D'Achile, L'italiano contemporaneo, Bologna 2003, pp. 246-250.

${ }^{8}$ F. Sabatini, Prove per l'italiano "trasmesso" [in:] Gli italiani trasmessi, Roma 1977, p. 18.

${ }^{9}$ P. D’Achile, op. cit., p. 250.

10 A. Masini, L'italiano contemporaneo e la lingua dei media, [in:] La lingua italiana e i mass media, a cura di I. Bonomi, A. Masini, S. Morgana, Roma 2003, pp. 28-29.

${ }^{11}$ D. Cardini, La televisione: linguaggio e modalità produttive, [in:] Manuale di sociologia della comunicazione, a cura di M. Livolsi, Bari 2002, p. 352.

${ }^{12}$ R. Setti, op. cit., p. 132.

13 R. Sardo, "Il discorso costruito": testualità e linguaggi della tv per bambini, [in:] Dall'Albero azzurro a Zelig: modelli e linguaggi della tv vista dai bambini, a cura di R. Sardo, M. Centorrino, G. Caviezel, Soveria Mannelli 2004, p. 126.

${ }^{14}$ Ivi, p. 129.

${ }^{15}$ A. Masini, op. cit., p. 37.
} 
vogliamo, quindi, dimostrare eventuali corrispondenze tra la lingua parlata e la lingua della televisione da questo punto di vista.

\section{La questione del congiuntivo}

La sostenibilità del congiuntivo nel parlato e la sua cosiddetta vitalità occupano una posizione particolare nella ricerca linguistica di oggi, sia sul piano sincronico che diacronico. La prevalenza dell'indicativo nei contesti più colloquiali non è un fenomeno recente (si nota perlomeno già nei capolavori della letteratura del Quattrocento $\left.{ }^{16}\right)$, ma la riflessione grammaticale a proposito è più recente: l'interesse per il congiuntivo risale alle grammatiche manzoniane ${ }^{17}$.

La questione della vitalità del congiuntivo nell'italiano contemporaneo rientra nella riflessione sulle varietà linguistiche italiane (diatoche, diastratiche, diafasche e diamesiche) che in realtà sono ancora in evoluzione ${ }^{18}$. La scelta modale viene quindi determinata da molteplici fattori extralinguistici. Sempre più interesse scientifico comporta l'opposizione tra scritto e parlato, tra i quali esiste una significativa sproporzione per quanto riguarda l'uso dei modi. L'uso meno frequente del congiuntivo è percepibile particolarmente nel parlato-parlato (il termine introdotto da Nencioni, 1976), caratterizzato come spontaneo e non sorvegliato $^{19}$. La modesta presenza del congiuntivo nel parlato è dovuta alla tendenza alla semplificazione morfosintattica, tipica delle conversazioni non sorvegliate ${ }^{20}$. L'alternanza tra $\mathrm{i}$ modi è una delle caratteristiche principali dell'italiano neostandard ${ }^{21}$ o dell'italiano contemporaneo in generale ${ }^{22}$. Similmente, la tendenza a introdurre l'indicativo invece del congiuntivo è più frequente nel parlato medio ${ }^{23}$, particolarmente nelle proposizioni dipendenti da

\footnotetext{
${ }^{16}$ L. Serianni, op. cit., p. 53.

${ }^{17}$ Ibidem.

${ }^{18}$ La necessità di analizzare qualunque fenomeno linguistico separatamente tra i confini di ogni variante viene menzionata già nelle grammatiche dell'Ottocento, nelle quali si evidenzia soprattutto la particolare rilevanza della opposizione tra scritto e parlato (Serianni 2011: 29)

19 G. Berruto, Varietà diamesiche, diastratiche, diafasiche, [in:] Introduzione all'italiano contemporaneo. La variazione e gli usi, a cura di A. Sobrero, Bari 2006, p 36.

${ }^{20}$ Ibidem, p, 70.

${ }^{21}$ F. Sabatini, op. cit., p. 166.

22 M. Dardano, P. Trifone, La nuova grammatica della lingua italiana con nozioni di linguistica, Bologna 1995, p. 363.

${ }^{23}$ F. Sabatini, op. cit., p. 166.
} 
verbi di opinione o di verbi di "sapere" e "dire" nelle frasi negative, interrogative indirette e relative restrittive ${ }^{24}$.

Nella riflessione grammaticale vengono sottolineati due aspetti del congiuntivo: da un lato, viene trattato come un esponente di incertezza e soggettività opposta al modo indicativo, dall'altro si riduce ad una scelta linguistica più elegante ${ }^{25}$. Battista Moretti osserva che per essere percepito come più sofisticato, lo stesso parlante cambierebbe il modo nelle frasi dipendenti, applicando il congiuntivo per esprimersi in maniera non solo più formale, ma anche raffinata ${ }^{26}$. Tuttavia, ridurre l'uso del congiuntivo a un elemento indispensabile della lingua ufficiale sarebbe un abuso e potrebbe provocare gli errori causati dall'ipercorrezione.

Anche se la semantica del congiuntivo è varia ed ha molte caratteristiche distinte, una dei più distaccati è la sua funzione di collegare la proposizione principale e subordinata e il rapporto sintattico fra queste ${ }^{27}$. La scarsa presenza del congiuntivo nella lingua parlata può essere debita precisamente alla sua sintassi, che si caratterizza dalla tendenza a una semplificazione morfosintattica, quindi la quantità delle frasi subordinate più piccola comparando con il numero delle frasi principali, la prevalenza della paratassi all'ipotassi e una gamma minore delle congiunzioni, rispetto allo scritto standard ${ }^{28}$. Tuttavia, non cambiano solo le lingue morte ${ }^{29}$ e analogicamente progredisce anche il parlato italiano, la varietà che "oggi è molto più compatta e diffusa di quel che fosse quarant'anni fa" ${ }^{30}$. La sua estensione si deve, tra gli altri, alla espansione dei diversi mezzi di divulgazione delle informazioni, tra i quali distacca la televisione.

\footnotetext{
${ }^{24}$ Anche se alcuni linguisti parlano della crisi del congiuntivo (si vedamo p. Es. Voghera, 1993 o Sensinisecondo cui "nell'italiano contemporaneo il congiuntivo è in crisi: incalzato dall'indicativo, che mira a usurparne il posto, esso è in lenta ma inesorabile decadenza"; 2005: 227), altri notano che la sua presunta scomparsa è piuttosto un frequente argomento della polemica tra neopuristi "amatori e difensori della Patria lingua" (Sgroi 2013: 9). La detta scomparsa viene sottolineata soprattutto dai "non specialisti", gli studiosi però "si premurano spesso, ove possibile, di relativizzare questa opinione, mettendo in luce talora esempi di vitalità attuale $<<$ del $\mathrm{C}$, talaltra esempi di sua "sostituzione« da parte dell'I già in scrittori e in epoche non sospetti" (Lombardi Vallauri 2003: 609).

25 S. C. Sgroi, Dove va il congiuntivo? Ovvero il congiuntivo da nove punti di vista, Torino, 2013, p. 9.

${ }^{26}$ G. Moretti Battista, L'italiano come prima o seconda lingua: forme, strutture, usi, Perugia 1996, p. 44.

${ }^{27} \mathrm{~K}$. Blücher, Il congiuntivo nell'italiano scritto contemporaneo, Roma 2018, p. 14.

${ }^{28}$ G. Berruto, op. cit., p. 17.

${ }^{29}$ S. C. Sgroi, op. cit., p. 4.

${ }^{30}$ L. Serianni, op. cit., p. 53.
} 


\section{Analisi - Metodologia}

La ricerca è basata sull'analisi quantitativa di un corpus del linguaggio televisivo (LIT) in comparazione con la banca dati della lingua parlata (CorDIC). Il parlato fornisce, infatti, un'ampia base per le osservazioni sui fenomeni linguistici, essendo non solo un piano linguistico separato, bensì una dimensione che "percorre le altre dimensioni di variazione e allo stesso tempo ne è attraversata" ${ }^{31}$; costituisce dunque un punto di riferimento affidabile anche per gli studi sull'uso dei modi, ed è governato inoltre da "[...] altre leggi rispetto allo scritto: ora è più ridondante, ora è molto più stringato"32.

Il LIT ${ }^{33}$, costituisce un'ampia banca dati testuale e audiovisiva, preparata dal Dipartimento di Italianistica dell'Università degli Studi di Firenze. Il sistema raccoglie un campione rappresentativo dell'italiano televisivo a partire dal 2006 e consta in 62 ore di parlato, 663.000 occorrenze e 39.000 forme $^{34}$. Visto che i dati vengono limitati alla rete RAI, la cui sede è situata a Roma, la varietà diatopica prevalente è romana. ( $w w w$.accademiadellacrusca.it/it/attivita/lessico-dellitalianotelevisivo-lit).

Come corpora dell'italiano parlato sono stati selezionati i corpora CorDIC LABLITA, sono stati progettati per offrire uno strumento di ricerca per il confronto tra le varietà scritta e orale (si veda Cresti e Panunzi ${ }^{35}$ ). CorDIC si articola in due sezioni indipendenti: il corpus scritto e il corpus parlato, ma l'analisi che segue si snoda esclusivamente nella seconda direzione. La parte del CorDIC-parlato è una raccolta delle trascrizioni del corpus di parlato spontaneo del Laboratorio Linguistico Italiano dell'Università di Firenze (LABLITA) ${ }^{36}$. La banca dati viene scelta tra gli altri numerosi corpora del parlato per la sua la comparabilità con LIT: la raccolta CorDIC contiene circa 500.000 parole suddivise in 200 campioni testuali.

Data la differenza tra il motore di ricerca in entrambe le banche dati, è stata adattata una metodologia duplice. Per il CorDIC, la banca dati è stata limitata al linguaggio parlato dialogico e monologico e tra diverse categorie di ricerca è scelta la di lemma, che permette di trovare tutte le forme verbali di un infinito esaminato. Ottenute le concordanze, i risultati sono stati filtrati e limitati a quelli, in cui nel primo o secondo posto dopo il verbo appare la congiunzione che.

\footnotetext{
${ }^{31}$ G. Berruto, op. cit., p. 19.

${ }^{32}$ L. Serianni, op. cit., p. 74.

${ }^{33} \mathrm{Il}$ corpus è consultabile sul portale www.italianotelevisivo.org.

${ }^{34}$ R. Setti, op. cit., p. 134.

${ }^{35} \mathrm{Si}$ veda anche il sito http://corporadidattici.lablita.it.

${ }^{36}$ Cresti E., Panunzi A., Introduzione ai corpora dell'italiano, Bologna 2013, p. 76.
} 
Al contrario, il sistema del corpus della lingua televisiva è meno automatizzato e per questo è scelto l'altro orientamento. Per mancanza della categoria di lemma, sono state ricercate tutte le forme verbali dell'indicativo e dei modi infiniti, accompagnate dal che. I risultati sono stati limitati al campo del testo parlato improvvisato.

Tenendo conto dell'uguaglianza delle forme dell'indicativo e congiuntivo presente della seconda persona singolare della prima coniugazione nonché della prima persona plurale, non verranno prese in considerazione tali concordanze. Sono tralasciate anche le frasi dove il nesso tra la proposizione reggente e quella subordinata viene ommesso.

L'elenco dei verbi ricercati nei corpora è stato preso dallo studio di Kolbjorn Blucher, presentato nel libro Il congiuntivo nellitaliano scritto contemporaneo (2018). Le concordanze vengono suddivise nei due gruppi: a) la proposizione oggettiva, b) la proposizione soggettiva. Per motivi di spazio nelle tabelle in continuazione vengono riportati solo i risultati per i verbi che nelle entrambe le banche dati hanno più di 5 concordanze. I risultati dello studio si dimostrano in dettaglio qui di seguito.

Tabella 1: Risultati del corpus dell'italiano parlato

\begin{tabular}{|c|c|c|}
\hline & Congiuntivo & Indicativo \\
\hline \multicolumn{3}{|c|}{ Completiva oggettiva } \\
\hline Volere & $39(90,7 \%)$ & $4(9,3 \%)$ \\
\hline Sperare & $51(87,9 \%)$ & $7(12,1 \%)$ \\
\hline Aspettare/attendere & $11(84,6 \%)$ & $2(15,4 \%)$ \\
\hline Augurare & $9(90 \%)$ & $1(10 \%)$ \\
\hline Chiedere & $8(100 \%)$ & 0 \\
\hline Lasciare & $5(100 \%)$ & 0 \\
\hline Preferire & $1(100 \%)$ & $10(83,3 \%)$ \\
\hline Convincere & $2(16,7 \%)$ & $84(45,7 \%)$ \\
\hline Suggerire & $1(100 \%)$ & $5(14,3 \%)$ \\
\hline Pensare & $100(54,3 \%)$ & $6(85,8 \%)$ \\
\hline Ritenere & $30(85,7 \%)$ & $48(30 \%)$ \\
\hline Sostenere & $1(14,2 \%)$ & 0 \\
\hline Credere & $112(70 \%)$ & $11(50 \%)$ \\
\hline Supporre & $5(100 \%)$ & $68(94,4 \%)$ \\
\hline Immaginare & $11(50 \%)$ & $392(91 \%)$ \\
\hline Capire & $4(5,6 \%)$ & $638(59,8 \%)$ \\
\hline Dire & $39(9 \%)$ & \\
\hline Insieme & $429(40,2 \%)$ & 0 \\
\hline
\end{tabular}




\begin{tabular}{|c|c|c|}
\hline \multicolumn{3}{|c|}{ Completiva soggettiva } \\
\hline Piacere/dispiacere & $12(75 \%)$ & $4(25 \%)$ \\
\hline Bisognare & $39(95,1 \%)$ & $2(4,9 \%)$ \\
\hline Bastare & $16(80 \%)$ & $4(20 \%)$ \\
\hline Sembrare & $64(74,4 \%)$ & $22(25,6 \%)$ \\
\hline Parere & $69(80,2 \%)$ & $17(19,8 \%)$ \\
\hline Risultare & $7(70 \%)$ & $3(3 \%)$ \\
\hline Succedere & 0 & $13(100 \%)$ \\
\hline Insieme & $207(76,1 \%)$ & $65(23,9 \%)$ \\
\hline
\end{tabular}

Tabella 2: Risultati del corpus dell'italiano televisivo

\begin{tabular}{|c|c|c|}
\hline & Congiuntivo & Indicativo \\
\hline \multicolumn{3}{|c|}{ Completiva oggettiva } \\
\hline Volere & $108(93,1 \%)$ & $8(6,9 \%)$ \\
\hline Sperare & $101(94,4 \%)$ & $6(5,6 \%)$ \\
\hline Aspettare/attendere & $14(82,3 \%)$ & $3(27,7 \%)$ \\
\hline Augurare & $20(90,9 \%)$ & $2(9,1 \%)$ \\
\hline Chiedere & $14(100 \%)$ & 0 \\
\hline Lasciare & $21(100 \%)$ & 0 \\
\hline Preferire & $6(100 \%)$ & 0 \\
\hline Convincere & $20(66,7 \%)$ & $10(33,3 \%)$ \\
\hline Suggerire & $8(100 \%)$ & 0 \\
\hline Pensare & $64(61 \%)$ & $41(39 \%)$ \\
\hline Ritenere & $30(100 \%)$ & 0 \\
\hline Sostenere & $7(36,8 \%)$ & $12(63,2 \%)$ \\
\hline Credere & $97(77 \%)$ & $29(33 \%)$ \\
\hline Supporre & $7(100 \%)$ & 0 \\
\hline Immaginare & $24(60 \%)$ & $16(40 \%)$ \\
\hline Capire & $4(6,9 \%)$ & $54(93,1 \%)$ \\
\hline Dire & $13(4,6 \%)$ & $267(95,4 \%)$ \\
\hline Insieme & $558(55,4 \%)$ & $450(44,6 \%)$ \\
\hline \multicolumn{3}{|c|}{ Completiva soggettiva } \\
\hline Piacere/dispiacere & $14(93,3 \%)$ & $1(6,7 \%)$ \\
\hline Bisognare & $23(95,8 \%)$ & $1(4,2 \%)$ \\
\hline Bastare & $13(72,2 \%)$ & $5(27,8 \%)$ \\
\hline Sembrare & $122(81,3 \%)$ & $28(28,7 \%)$ \\
\hline Parere & $69(70,4 \%)$ & $29(29,6 \%)$ \\
\hline Risultare & $11(84,6 \%)$ & $2(25,4 \%)$ \\
\hline Succedere & 0 & $9(100 \%)$ \\
\hline Insieme & $252(77,1 \%)$ & $75(22,9 \%)$ \\
\hline
\end{tabular}


L'analisi dei corpora conferma una tendenza, caratteristica per l'italiano parlato medio, a introdurre l'indicativo invece del congiuntivo, particolarmente in proposizioni rette da verbi di opinione ${ }^{37}$. La situazione particolare si dimostra per il verbo dire. La maggior parte delle concordanze nel congiuntivo, sono le frasi con valore di modalità deontica, con scarsi esempi delle proposizioni rette dal dire in negativo. La naturale prevalenza delle frasi nell'indicativo è collegata con le costruzioni tipiche per la modalità epistemica.

Nelle concordanze analizzate del LIT l'indicativo appare soprattutto nelle frasi rette dal verbo in seconda persona singolare (fenomeno considerato proprio per la lingua colloquiale ${ }^{38}$ ) e in prima persona singolare del presente. Alcuni verbi, tali come sperare, sostenere, immaginare etc, reggono prevalentemente le costruzioni nel futuro semplice, che suggerisce, tenendo conto del suo valore modale, che l'emittente, dopo questi verbi tende a sottolineare la sfumatura del dubbio, supposizione o deduzione. Per quanto riguarda l'altro gruppo delle concordanze, rette da consentire/acconsentire, permettere, rischiare etc, entrambe le lingue preferiscono l'uso del pronome di + infinitivo, nel posto del "che" con indicativo/congiuntivo.

Grazie alle statistiche consultabili per ogni frase nel corpus LIT, è stato possibile definire il contesto televisivo delle concordanze. Tra i programmi più frequenti si potrebbe elencare i telegiornali, reality shows e le trasmissioni sportive.

\section{Conclusioni}

I risultati della ricerca dimostrano una certa somiglianza tra il linguaggio televisivo e il parlato. Questa similitudine si nota, tra l'altro, dalla modesta quantità o da una completa mancanza degli stessi verbi nei due corpora, i quali verbi sono propri piuttosto della lingua scritta (appaiono nell'analisi del corpus dell'italiano scritto, condotto da Blucher). Significativo è anche il verbo succedere, che appare in entrambi i corpora solo all'indicativo (nello studio di Blucher ci sono due esempi di succedere al congiuntivo).

Malgrado le suddette similarietà, esiste una significativa differenza nell'uso del congiuntivo in entrambi i sistemi. Per più della metà dei verbi, il congiuntivo è più frequentemente nel linguaggio televisivo. La differenza più notevole riguarda soprattutto i verbi di opinione: pensare, ritenere, sostenere, credere, sembrare ma anche di emozioni e volontà: volere, sperare piacere. Il verbo convincere

${ }^{37}$ F. Sabatini, op. cit., p. 166.

${ }^{38}$ M. Dardano, P. Trifone, op. cit., p. 388. 
al congiuntivo presenta differenze più significative tra le due banche dati (nel parlato: $16,7 \%$, nel televisivo: $66,7 \%$ ). Inoltre, anche se il parlato trasmesso cerca di avvicinarsi al parlato-parlato, senza dubbio la variante televisiva è sempre meno spontanea e condizionata dalle circostanze. La frequente presenza dell'indicativo nel parlato, collegato alla scelta linguistica più colloquiale ${ }^{39}$ non è del tutto marginale. Ciononostante, tale spontaneità dell'espressione verbale viene ancora vista nella televisione, per così dire, con una certa timidezza. Sarebbe quindi più adeguata la metafora proposta da Masini che compara la lingua dei mass media piuttosto a un raggio che a uno specchio.

I risultati dell'analisi dimostrano una sottile disproporzione tra l'uso dell'indicativo nella lingua parlata e nel linguaggio televisivo che risulta sempre più ufficciale rispetto al parlato non sorvegliato. Questa asimmetria potrebbe essere dovuta alla funzione sintattica del congiuntivo (usato soprattutto nelle frasi subordinate molto meno frequenti nel linguaggio televisivo). È importante anche la specificità dell'italiano televisivo: di frequente, il parlante comincia la frase con il verbo che richiederebbe il congiuntivo, tuttavia il suo comunicato viene interrotto dal suo interlocutore e perciò finisce la stessa frase scegliendo l'indicativo.

In ogni modo, dai risultati dell'analisi sorge un panorama perlomeno ottimistico: la tesi sulla presunta scomparsa del congiuntivo nel parlato sembra almeno in questo caso - esagerata. In ogni caso che abbiamo analizzato, il numero delle frasi con il congiuntivo supera il $60 \%$ delle concordanze, il che dimostra che il congiuntivo è ancora ben presente nella lingua trasmessa dalla televisione, come anche nel parlato non sorvegliato. Inoltre, visto che tra gli obiettivi del linguaggio televisivo c'era , tra gli altri, "far entrare nelle case e nelle orecchie di tutti gli italiani i suoni uniformi di una lingua il più possibile esente dalle inflessioni locali e regionali" ${ }^{40}$, all'inizio il discorso dei media era un esempio da seguire per gli utenti della lingua. Tuttavia, la televisione di oggi (in confronto con i primi programmi) è diventata molto più spontanea, pertanto non si può negare l'influsso del parlato alla lingua della TV. Entrambi i sistemi rimangono, quindi, in una vera e propria simbiosi.

\footnotetext{
${ }^{39}$ L. Serianni, op. cit., p. 31.

${ }^{40}$ R. Setti, op. cit., p. 131.
} 


\section{Bibliografia}

Berruto G. (2006). Varietà diamesiche, diastratiche, diafasiche, [in:] Introduzione all'italiano contemporaneo. La variazione e gli usi, a cura di A. Sobrero, Bari, pp. 3-36.

Blücher K. (2018). Il congiuntivo nell'italiano scritto contemporaneo, Roma.

Cardini D. (2002). La televisione: linguaggio e modalità produttive, [in:] Manuale di sociologia della comunicazione, a cura di M. Livolsi, Bari, pp. 351-396.

Cresti E., Panunzi A. (2013). Introduzione ai corpora dellitaliano, Bologna.

D'Achile P. (2003), L'italiano contemporaneo, Bologna.

Dardano M., Trifone P. (1995). La nuova grammatica della lingua italiana con nozioni di linguistica, Bologna.

De Mauro T. (1979). Storia linguistica dell'Italia Unita, Bari.

Della Valle V., Patota G. (2014). Viva il congiuntivo!, Milano.

Klímová E. (2011). Il congiuntivo nell'italiano scritto e parlato, "Echo des Etudes Romanes" (2011), 2, pp. 45-55.

Lavinio C. (1986). Fiaba popolare, oralità, vernacolo, [in:] Interni e dintorni del Pinocchio. Atti del Convegno Folkloristi italiani del tempo del Collodi, a cura di P. Clemente e M. Festa, Montepulciano.

Lombardi Vallauri E. (2003). Vitalità del congiuntivo nell'italiano parlato, [in:] Italia linguistica anno Mille, Italia linguistica anno Duemila: Atti del XXXIV Congresso Internazionale di Studi della Società di Linguistica Italiana, Roma, pp. 66-98.

Masini A. (2003). L'italiano contemporaneo e la lingua dei media, [in:] La lingua italiana e i mass media, a cura di I. Bonomi, A. Masini, S. Morgana, Roma, pp. 28-53.

Moretti Battista G. (1996). L'italiano come prima o seconda lingua: forme, strutture, usi, Perugia.

Sabatini F. (1985). L'italiano dell'uso medio: una realtà tra le varietà linguistiche italiane, [in:] Gesprochenes Italienisch in Geschichte und Gegenwart, a cura di E. Holtus, Tubingen, pp. 82-123.

Sabatini F. (1977). Prove per l'italiano "trasmesso" [in:] Gli italiani trasmessi, Roma, pp. 11-30.

Sardo R. (2004). "Il discorso costruito": testualità e linguaggi della tv per bambini, [in:] Dall'Albero azzurro a Zelig:modelli e linguaggi della tv vista dai bambini, a cura di R. Sardo, M. Centorrino, G. Caviezel, Soveria Mannelli, pp. 126-143.

Schneider S. (1999). Il congiuntivo tra modalità e subordinazione: uno studio sullitaliano parlato, Roma.

Serianni L. (2011). Prima lezione di grammatica., Roma.

Setti R. (2012). Primi sondaggi sul lessico televisivo dei programmi RAI, [in:] I linguaggi dei media, a cura di S. Stefanelli, V. Saura, Firenze, pp. 131-149.

Sgroi S. C. (2013). Dove va il congiuntivo? Ovvero il congiuntivo da nove punti di vista, Torino.

Widłak S. (2002). Gramatyka języka włoskiego, Varsavia. 
http://www.accademiadellacrusca.it/it/attivita/lessico-dellitaliano-televisivo-lit (data di consultazione: 08.01.2019)

http://www.italianotelevisivo.org (data di consultazione: 08.01.2019)

http://corporadidattici.lablita.it/ (data di consultazione: 08.01.2019) 